\title{
La política nacional/internacional de las telecomunicaciones en
} América Latina

\author{
Óscar Plaza
}

El presente trabajo exanina las políticas de telecomunicaciones aplicadas en América Latina en las dos últimas décadas. De acuerdo con la particular lectura que se hace de estas políticas, ellas no serian el resultado lineal de las politicas económicas en práctica. En consecuencia, el estudio propone que la adopción e implenentación de las políticas nacionales de telecomunicación en América Latina son el resultado de un complejo acoplaniento entre las politicas económicas vigentes y desarrollos tecnológicos y políticas internacionales en materia de telecomunicaciones. El encuentro de estos componentes habria creado el escenario politico en que han debido desarrollarse las telecomunicaciones en la región.

\section{Introducción}

Este trabajo intenta analizar el emergente sistema de telecomunicaciones dada la evolución de las políticas nacionales e internacionales en materia de telecomunicaciones. Utilizamos la experiencia latinoamericana como caso de estudio para nuestro análisis y discusión.

Latinoamérica es un caso exitoso de desarrollo de las telecomunicaciones. En el lapso de pocos años, ha hecho la transición desde un sistema anticuado y de escasa cobertura a uno tecnológicamente avanzado y que penetra rápidamente todas las regiones geográficas y estratos sociales.

Mientras esto ocurre, la tendencia mundial en materia de políticas de telecomunicaciones tiende hacia el abandono de la propiedad y administración de la industria por el Estado en favor de una política de mercado. El caso latinoamericano pareciera avalar el éxito de esta tendencia. En efecto, el desarrollo de las telecomunicaciones en Latinoamérica ocurre en paralelo con un énfasis en políticas de mercado para las economías en general.

Sin embargo, este marco de política económica general nos deja con poca comprensión de las políticas de telecomunicaciones específicas que han ocurrido durante el proceso de privatización y liberalización de la 
industria de telecomunicaciones. Nuestra propuesta sostiene que el desarrollo exitoso de las telecomunicaciones en Latinoamérica puede ser mejor entendido si analizamos en retrospectiva la forma en que las políticas nacionales se han acoplado a políticas internacionales y desarrollos tecnológicos en materia de telecomunicaciones. Este acoplamiento ha sido confuso y complicado, pero creemos que la interacción entre estos procesos y de los respectivos grupos de interés ha creado el escenario político en el cual ha sido posible el desarrollo de las telecomunicaciones.

Para los efectos de nuestra proposición, tratamos de evaluar las políticas públicas que han estado detrás del desmantelamiento del antiguo sistema de telecomunicaciones. Tratamos de identificar hechos que han facilitado o impedido políticas similares en otras regiones geográficas. Dentro de estos hechos, sostenemos que las capacidades técnicas de los nuevos sistemas de telecomunicaciones son un buen predictor de las actitudes políticas y las políticas que se adoptan con respecto a la industria. Explorando este tipo de aspectos, tratamos de dar fundamento al análisis de las políticas de telecomunicaciones en Latinoamérica.

\section{La tecnología}

Durante las tres últimas décadas hemos asistido a la transición desde la transmisión de voz vía cables pareados de cobre a la transmisión digital simultánea de voz, imagen, y datos. Esta transición ha sido dramáticamente rápida y está todavía en plena fase de aceleración. Al mismo tiempo, el decrecimiento en costo y tamaño, la mayor velocidad, capacidad y versatilidad del computador lo han transformado de una máquina calculadora a un componente básico de la red de telecomunicaciones extendiendo exponencialmente la capacidad de servicio de una red.

Hoy en día, un sistema de telecomunicaciones moderno es una tecnología que combina varias innovaciones, por ejemplo, la conmutación numérica digital y la transmisión vía fibra óptica o vía satelital. Todas éstas son innovaciones tecnológicas en evolución lo cual transforma la tecnología de telecomunicaciones en una tecnología en permanente y rápido proceso de cambio. El desarrollo tecnológico en telecomunicaciones puede ser clasificado en tres grandes áreas:

1) conmutación en la red pública, esto es, equipos que permiten la conexión entre puntos terminales de la red y coordinan la red de telecomunicación en su totalidad; 
2) terminales, los cuales son equipos conectados a los puntos terminales de la red pública, equipos tales como simples teléfonos, telecables, facsímiles, computadores y modems, o incluso conmutadores locales, y

3) transmisión, esto es, equipos que transportan las señales desde las estaciones terminales a los centros de conmutación.

Prácticamente todo este desarrollo tecnológico es generado en países pertenecientes a la OCDE. Cerca del $30 \%$ del total de los equipos de telecomunicaciones son equipos de conmutación y estos son producidos casi en su totalidad por compañías de países de la OCDE. Y la conmutación es la viga maestra de la gestión tecnológica y económica de un sistema de telecomunicaciones. Sin una infraestructura electrónica de conmutación, no hubieran sido posible avances como la división espacial de transmisión de la década de los 60 , el sistema de conmutación digital total de los 70, y el acceso y comercialización de información sin restricción de tamaño o ubicación del usuario (Roobeck, 88).

Debido al rápido progreso en programación computacional, procesamiento de datos, tecnología de transmisión, y capacidad de los terminales, la distinción entre conmutación central y conmutación local está perdiendo sentido, especialmente cuando se trata de redes de alta densidad. Consecuentemente, la importancia relativa de la capacidad de conmutación central de una red de telecomunicaciones está declinando, lo cual abre varias alternativas de administración técnica y comercial de la red.

La conmutación digital y la transmisión óptica son tecnologías cada día más esenciales en un sistema de telecomunicaciones. Los sistemas modernos de telecomunicaciones poseen la capacidad de ofrecer una variada gama de servicios a una variada gama de usuarios. Estos servicios no son posibles con las tecnologías electromecánicas de sistemas de telecomunicaciones anteriores.

En definitiva, las nuevas tecnologías en telecomunicaciones no sólo son tecnológicamente superiores sino económicamente más eficientes. A igual servicio, las nuevas tecnologías son de menor costo. Adicionalmente, dada la capacidad de mejor $y / o$ nuevos servicios, el potencial de expansión de la industria de las telecomunicaciones es gigantesco.

La transición desde la tecnología de conmutación electromecánica a la electrónica se ha visto afectada por dos factores específicamente tecnológicos. Primero, la instalación de equipos de conmutación electromecánicos se dio en medio de expectativas de expansión de largo plazo. 
Una vez que la infraestructura estuviera instalada, nuevas líneas de transmisión podían ser agregadas a un muy bajo costo marginal alcanzando la capacidad máxima del sistema en cinco o diez años. La infraestructura total se diseñó para un servicio de vida útil de alrededor de cincuenta años. Por lo tanto, países de industrialización temprana pudieron razonablemente desarrollar una amplia infraestructura electromecánica y esperar amortizar costos en el largo plazo. Países no industrializados y/o con un pequeño nivel absoluto de densidad telecomunicacional enfrentaban costos de infraestructura prohibitivos. Segundo, la integración de los nuevos sistemas de conmutación electrónica dentro de una infraestructura electromecánica existente es mucho más compleja técnicamente y más costosa económicamente que construir una red electrónica digital desde tabla rasa.

Dada las características de una red de telecomunicaciones moderna, técnica y económica resulta entonces más conveniente el completo reemplazo de una infraestructura preexistente que el intento de modernizarla gradualmente, aunque financieramente esto resulte oneroso.

Esta misma característica determina que países que tenían una baja densidad de telecomunicaciones y países con una infraestructura muy desarrollada, están enfrentando estructuras de costos e incentivos para inversión de naturaleza muy divergente.

\section{Implicaciones de política}

Las características técnicas de la tecnología de telecomunicaciones contemporánea tiene efectos en su difusión internacional. Estos efectos generan tres importantes consecuencias. Primero, las probabilidades de rápida difusión son mucho mayores en países con alto crecimiento de la demanda, dada la gran capacidad de expansión de conexiones de los modernos sistemas de telecomunicaciones. Segundo, la difusión será probablemente más rápida en países de baja densidad en telecomunicaciones dado el menor monto de costos hundidos en infraestructura previa, al momento de introducir la innovaciones en telecomunicaciones. Tercero, la difusión tenderá a ser mayor en países con capacidad de inversión en telecomunicaciones.

Pareciera ser el caso que la tecnología de telecomunicaciones moderna ofrece un muy buen ejemplo de oportunidad de salto tecnológico (Plaza, 97). Los primeros adoptantes tienden a ser países que no han completado el desarrollo de una infraestructura de telecomunicaciones 
moderna y por tanto están en una posición técnica y financiera más flexible para incorporar nueva tecnología. Se exceptuarían de esta norma países muy avanzados, en los cuales la motivación principal es el liderazgo tecnológico. En todo caso, países relativamente atrasados tienen costos de adopción mucho más bajos dado que no están amarrados a una estructura de instalaciones, de proveedores, de diseño, que deban ser reemplazados antes de que las innovaciones en tecnología de telecomunicaciones pueda ser implementada. Además, estos países pueden beneficiarse de continuas bajas en los precios de tecnologías avanzadas, menores costos de mano de obra, más y mejor información acerca de proveedores internacionales, y mayor apertura a la importación dada la inexistencia de proveedores nacionales a los cuales apoyar.

Países de atraso relativo con economías emergentes pueden beneficiarse con una alta tasa de inversión en la industria de telecomunicaciones (Storper, 98). Riesgos asociados a irreversibilidad de inversiones son mucho menores en países seguidores que en países con alta inversión en bienes de capital ya ejecutada. Adicionalmente, países recién llegados al mundo de las telecomunicaciones modernas no tienen que preocuparse de la amortización y depreciación de la infraestructura previa.

La velocidad de difusión entre los nuevos adoptantes de tecnología telecomunicacional varía considerablemente. Depende de factores tales como el clima de negocios, tasas de interés, rentabilidad comparada entre nuevos bienes de capital y los ya existentes, los resultados obtenidos con la nueva tecnología en otros países, la cantidad y calidad de infraestructura telecomunicacional existente, disponibilidad de recursos humanos calificados, disponibilidad de servicios técnicos, nivel de salarios, acceso y costo financiero. En cuanto a costo de la mano de obra en el país adoptante, a mayor costo más incentivo para el desarrollo de las telecomunicaciones, dado el alto impacto de esta industria en la productividad de la mano de obra en el sector servicios, que es, a su vez el sector más dinámico en términos de empleo.

Un factor decisivo de política para explicar la situación actual de la industria de telecomunicaciones, es la tendencia hacia una mayor libertad de comercio en telecomunicaciones en los mercados de las economías más desarrolladas. Este movimiento comenzó con el mandato de desmembramiento de la compañía AT\&T de los Estados Unidos y se expandió por todo el mundo con las llamadas políticas de privatización, liberalización, y desregulación de la industria de telecomunicaciones. Otro factor clave para la industria ha sido el explosivo crecimiento de la demanda que ha seguido a las innovaciones tecnológicas en telecomunica- 
ciones. Este fenómeno ha permitido a su vez un extraordinario crecimiento en la capacidad de manufacturación de equipos de telecomunicaciones. Como resultado del incremento cuantitativo y cualitativo de la oferta de equipos de telecomunicaciones y de la mayor libertad de comercio en economías de países claves, se ha observado el desarrollo de un mercado extremadamente competitivo de la industria de telecomunicaciones como un todo a nivel mundial.

Los tradicionales gigantes de las telecomunicaciones han estado enfrentando un verdadero asalto en los otrora estables y seguros mercados de consumidores. Los atacantes son empresas emergentes que utilizan como arma de combate nuevas tecnologías telecomunicacionales, tales como la fibra óptica de banda ancha en lugar de la relativamente estrecha vía del cable de cobre, paquetes de conmutadores digitales en lugar de tradicionales circuitos, y red de protocolos en Internet. Para estas nuevas empresas la era de los mercados diferenciados entre telefonía y transmisión de datos terminó, sólo hay un mercado combinado de transmisión de voz/datos/imagen. A nivel nacional, los tradicionales gigantes están perdiendo crecientes porciones del mercado a manos de estos nuevos rivales que se inician con tecnologías avanzadas y, por tanto, con costos de servicios equivalentes iguales a un cuarto o un quinto de los costos tradicionales. En el mercado internacional, estos gigantes, a pesar de alianzas estratégicas, su experiencia en gestión de redes, y su conocimiento de contra estrategia, tienen serias dificultades en contrarrestar la agilidad empresarial, la rapidez de decisiones, y el espíritu de riesgo de estas compañías que se generan a partir de recientes posibilidades tecnológicas. Mientras la industria de telecomunicaciones se asemeje cada vez más a la de computación, con rápida evolución tecnológica y con oferta de variados servicios, los tradicionales gigantes de las telecomunicaciones, sean de propiedad estatal o privada, tendrán más dificultades en captar el incremento explosivo del tráfico telecomunicacional.

Un alto grado de consenso técnico se ha logrado en la industria de telecomunicaciones, concerniente a la necesidad de construir Servicios Integrados de Redes Digitales (ISND, la sigla en inglés). Éste es un diseño de redes que permite acceso a un considerable número y variedad de terminales, teléfonos, télex, facsímil, correo electrónico, computadores, etc., proveyendo conectividad entre ellos de manera que puedan intercambiar tráfico de voz/dato/imagen simultáneamente. Esta tecnología es un paso gigante hacia la globalización de las telecomunicaciones, un objetivo al cual se subscriben prácticamente todos los países del mundo. Sin embargo, aun dada esta tecnología, existen muchos proble- 
mas políticos y de políticas de telecomunicaciones por resolver que son pre-requisitos para acortar la brecha del desequilibrio internacional en materia de desarrollo en telecomunicaciones. Una primera consideración es la necesidad de reajustar las prioridades de inversión en países en desarrollo, en vista de la evidencia de que un eficiente y bien mantenido sistema de telecomunicaciones es un sistema de carreteras, teniendo en mente, además que en países desarrollados el sector de la información contribuye entre un tercio y un cuarto del Producto Nacional Bruto.

\section{Las telecomunicaciones en Latinoamerica}

Al hablar de este fenómeno en la región el principal error que podríamos cometer seria el agrupar a los países de América Latina en un todo. La única manera de entender las sutiles diferencias culturales y socioeconómicas y la forma en que éstas afectan el potencial de crecimiento de la industria de telecomunicaciones, es mediante un riguroso análisis en terreno. Sin embargo, diferencias aparte, existe un común denominador en cuanto al interés en telecomunicaciones en Latinoamérica: una aguda necesidad de mejor conectividad. Dado el lánguido desarrollo que tuvo la industria de las telecomunicaciones y el deplorable estado de la red tradicional, es difícil imaginar a un empresario, por ejemplo, genuinamente interesado en la revolución tecnológica que significan las nuevas tecnologías telecomunicacionales.

Sin embargo, hoy en día Latinoamérica tiene, en promedio, un rezago de no más de tres años con respecto a países desarrollados, en cuanto a introducción de innovaciones tecnológicas en telecomunicaciones. Generalmente, los países latinoamericanos contratan la manufacturación y diseño de sus sistemas de telecomunicaciones con empresas de países desarrollados. Con frecuencia, estas compañías descubren que la instalación y gestión de sistemas de telecomunicaciones en esta región requiere de ajustes inesperados. Hábitos culturales obligan a cambiar patrones de gestión. Por ejemplo, los latinoamericanos acostumbran a acostarse tarde y a conversar hasta tarde; resultado: una alta demanda por servicios de telecomunicaciones pasada la media noche. Otro descubrimiento que sorprendió a las compañías de países desarrollados es el altísimo uso del teléfono celular en Latinoamérica. Mucho más si se considera que el precio del servicio es comparable a aquel vigente en países desarrollados. Dada la diferencia en nivel de ingreso medio, el 
costo relativo del servicio es muchísimo más caro que en el mundo desarrollado, y a pesar de esto el nivel de consumo es mucho más elevado.

No obstante el extraordinario crecimiento de la industria de telecomunicaciones en América Latina, las compañías operadoras se ven afectadas por el elevado costo de importación de equipos. Las tarifas de importación suelen llegar al 50\%. Pero, más resienten las barreras no tarifarias, como el tener que apuntalar a una fábrica de atornilladores, a fin de satisfacer regulaciones locales de manufacturación. Algunos países todavía usan un enfoque de empleo con respecto a la importación de equipos de telecomunicación, otros prefieren elevar las tarifas de importación. Otros países que no elevan excesivamente las tarifas, elevan en gran medida el precio de importación imponiendo una elevada tasa al valor agregado, tan alta como $30 \%$.

Los requerimientos técnicos de importación son generalmente mínimos en Latinoamérica. Por ejemplo, basta la aprobación del producto por la Comisión de Comunicaciones Federales de los Estados Unidos. Los canales de distribución de equipos son generalmente controlados en forma estricta por los manufacturadores. Existen dos razones para esta conducta. Inicialmente, los manufacturadores son los únicos que realmente conocen el producto y por tanto capaces de venderlo para el uso apropiado. Los manufacturadores no desean que el producto se desprestigie debido a un uso inadecuado. Pero, una vez introducido el producto en el mercado, el peligro de copia fraudulenta genera una segunda razón de control de los canales de distribución. Como resultado, muchos distribuidores latinoamericanos representan una sola marca de equipos de telecomunicación. En algunos mercados, a los distribuidores les está permitida la venta de otros productos, tales como equipos electrónicos. En algunos países, las compañías operadoras tienen sus propios distribuidores exclusivos y los ingresos provienen como participación del servicio de telecomunicaciones de la operadora. Esta modalidad tiene como una de sus motivaciones el obviar regulaciones que afectan el negocio de distribución.

La estabilidad monetaria constituye un elemento de preocupación adicional para las compañías operadoras de telecomunicaciones en América Latina. Inflación, inestabilidad cambiaria, y controles cambiarios son factores que ponen en jaque el sistema de determinación de precio de servicios. Y si se trata de servicios de conexión con el extranjero, el problema se complica más. Por esta razón, en muchos países, los cargos por estos últimos servicios se hacen en dólares para ser pagados en la misma divisa. 


\section{Efectos políticos}

Originalmente, sectores políticos y empleados de las empresas de telecomunicaciones de América Latina, se negaban a ver el negocio involucrado en la industria de telecomunicaciones. Ellos percibían la industria como una empresa de servicio social. En época tan reciente como una década atrás, en muchos países de la región se pensaba que la industria de telecomunicaciones debía ser un monopolio, y no sólo un monopolio, sino uno estatal.

El pensamiento político con respecto a la industria de telecomunicaciones ha cambiado rápidamente (Boeker, 93). Hoy en día, prácticamente todos los gobiernos latinoamericanos se dan cuenta de que el Estado no es capaz de administrar la industria en forma apropiada, satisfacer la demanda creciente, proveer financiamiento, y mucho menos, evaluar el desarrollo de la tecnología moderna y sus implicaciones. Paralelamente, en un esfuerzo por mejorar las condiciones económicas, la política económica de la región se ha inclinado más hacia la apertura comercial que al tradicional proteccionismo.

La política de transición desde una empresa estatal de telecomunicaciones hacia compañías privadas competitivas ha enfrentado dos obstáculos principales. Primero, liberar la empresa estatal de sus beneficiarios, y segundo, atraer compradores para la licitación de la empresa. Los principales beneficiarios directos de las empresas han sido los empleados mismos. Sindicatos de trabajadores y administradores de las empresas temen perder empleos, paga, y sobre todo influencia. Esta última es también la que temen perder sectores políticos ligados al sector laboral. Y, por supuesto, estos beneficiarios directos se han opuesto a la privatización de las empresas de telecomunicaciones. Empresarios de todo tipo y nivel de actividades generalmente han sido promotores de la privatización con la esperanza de ver más y mejores servicios. Los usuarios residenciales son más ambivalentes al respecto porque por un lado esperan más y mejor servicio, pero por otro temen tarifas más elevadas (Ravi, 96).

Además de convencer a una reluctante ciudadanía de los beneficios de la privatización de la industria de telecomunicaciones, los gobiernos latinoamericanos se han debido esmerar para atraer potenciales compradores. En general, no ha sido difícil vender las empresas del rubro. Dentro de las compañías estatales, éstas han estado relativamente mejor administradas y ha existido un exceso de demanda por sus servicios, literalmente con millones de solicitudes de conexión en listas de espera y 
creciente demanda por nuevos servicios telecomunicacionales. Las empresas han estado groseramente sobredotadas de personal, pero este problema de alguna manera se ha paliado con la proyectada rápida expansión de las empresas, alrededor de $12 \%$ por año en promedio (Ravi, 96:83). Con el fin de darle mayor atractivo comercial a las empresas, los gobiernos normalmente han elevado drásticamente la tarifa de los servicios antes de la privatización y han otorgado una licencia monopólica por un número fijo de años, en promedio alrededor de seis años.

La campaña promocional de los gobiernos latinoamericanos ganó gran interés internacional. Dado que los mercados nacionales de las grandes compañías de telecomunicaciones estaban creciendo mucho más lentamente que el mercado latinoamericano, muchas empresas multinacionales de telecomunicaciones se interesaron en posicionarse en este nuevo mercado de gran potencial. Además, estas empresas se dieron cuenta que los ingresos generados en sus respectivos mercados domésticos dejaron de estar asegurados, dada la férrea competencia de parte de las nuevas corporaciones emergentes debido a los cambios tecnológicos. Fue ésta la principal razón por la cual estos gigantes de las telecomunicaciones tomaron en serio el desafío de transformarse en portadores globales, capaces de servir a cualquiera en cualquier parte del mundo. Fueron ellos lo que acuñaron la hoy en día común expresión "Global Business". Sin estos cambios de política internacional de dichas multinacionales, es muy dudoso que estas compañías se hubieran interesado en las empresas de telecomunicaciones estatales de América Latina únicamente debido al buen trabajo de promoción de parte de los gobiemos de la región.

En la medida que el concepto de privatización ha ganado popularidad, ha adquirido también una variada gama de significados. Para algunos, significa toda una concepción de política económica, para otros, algo más que una específica transacción comercial. Para darle un carácter más significativo y operacional, el concepto debe entenderse como el completo proceso a través del cual el Estado renuncia al control de una empresa. Este proceso incluye las fases de preparación, privatización propiamente tal, y post-privatización. La forma práctica del proceso varía mucho. Puede tomar la forma de un remate, venta negociada, u oferta de compra. El Estado puede optar por simplemente capitalizar la firma distribuyendo títulos. Títulos que pueden ser comercializados en el mercado u ofrecidos en preferencia a los empleados de la empresa. Cabe hacer notar que también el Estado puede optar por una privatización parcial de la empresa, es decir, no entregando el control del ciento por 
ciento de la empresa. En este caso, se puede optar por un "joint venture", un contrato de arrendamiento, un contrato de administración, u otra modalidad (Ernst and Young, 94).

Los objetivos de quienes propician la privatización de las telecomunicaciones varían mucho. Se tiende a pensar que las razones de Estado son principalmente financieras. Esto es, deshacerse de una empresa que genera un continuo drenaje de los recursos presupuestarios, o bien inyectar un fuerte monto de capital al presupuesto de inversiones, o las dos razones en conjunto. Pero, esto es sólo parte de la historia. Al menos en América Latina, el proceso de privatización en general, y en telecomunicaciones en particular, ha sido requerido por instituciones financieras internacionales, empresarios multinacionales, e incluso por gobiernos extranjeros en foros internacionales o en encuentros bilaterales. A nivel nacional, la privatización ha sido promovida como una necesidad de la integración en el mercado global, como simple instrumento de eficiencia técnico/económica, o como parte de una concepción global de una economía de mercado.

La correlación entre privatización y eficiencia ha sido cuestionada por algunos teóricos (Rowthorn y Ha-Joon, 93). Una razón teórica para la privatización es la llamada "teoría del demandante residual". De acuerdo a esta teoría, la propiedad privada de una empresa es preferible, porque un demandante residual de ingreso genera valor adicional a la empresa, lo cual es precisamente lo que mantiene a los poseedores de títulos de la misma con incentivo para seguir como tales. Los críticos de esta teoría señalan que si la privatización significa vender títulos a diversos grupos, los cuales se supone, monitorean y toman decisiones, éstos estarán en una muy mala posición para cumplir su rol pues tendrán menos información y conocimiento que los actuales ejecutivos de la empresa. La contra respuesta a esta crítica, es que quien monitorea a la empresa no son los propietarios de títulos, sino el mercado. Si la empresa no funciona bien, los clientes desaparecerán, los ingresos disminuirán, y los poseedores de título los venderán. Sin embargo, debemos asumir varios supuestos al asignar al mercado el papel de regulador. Primero, si bien es cierto que los clientes pueden irse con otro proveedor, ese no es siempre el caso en telecomunicaciones, donde es posible que compañías, aun privatizadas, puedan mantener un mercado cautivo. Segundo, los precios del mercado de valores no se basan solamente en eficiencia de las empresas. Aun en mercados de valores de países desarrollados, no existe adecuada correlación entre precios y eficiencia, menos entonces en economías emergentes. 
Otra teoría utilizada para justificar la privatización es la llamada "teoría del conocimiento disperso". La teoría sostiene que dada la imposibilidad de captar todo el conocimiento económico dentro de la jerarquía de un sistema de planificación, las decisiones serán más eficientes si se las deja al mercado. Los críticos de la teoría sostienen que si bien el mercado puede ser un buen asignador de recursos, el uso de conocimiento disperso es difícil, sea que se trate de empresas privadas o estatales. La contra respuesta sostiene que la propiedad privada en si no determina mejores decisiones, sino que la propiedad privada expone a la empresa a la competición y a duras restricciones presupuestarias y esto sí que hace la diferencia.

El concepto de privatización tiene una connotada carga ideológica que genera reacciones muy distintas según sean los grupos de interés a nivel nacional o en relación a casos específicos. La Constitución Política de Brasil, por ejemplo, establece que el servicio de teléfonos puede ser otorgado sólo por empresas estatales. A fin de avanzar un proyecto de privatización, sin tener que ir cuesta arriba con un cambio constitucional, el gobierno ha tenido que recurrir a una sibilina interpretación de la disposición constitucional, sosteniendo que las telecomunicaciones no son para el público en general, sino para aplicaciones específicas.

Generalmente, remates y ofertas selladas, o simples ofertas han sido más aceptables para la opinión pública que las negociaciones de venta. Esto es especialmente el caso, cuando el gobierno decide otorgar títulos preferenciales a ciertos grupos de personas, por ejemplo, administradores de la empresa a privatizar. No obstante todas las medidas que los gobiernos han tomado para garantizar la transparencia en las privatizaciones, siempre existe una gran secuela de reclamaciones cuando se privatiza una empresa de telecomunicaciones. En México, por ejemplo, el cuestionamiento llegó a tal nivel que hubo necesidad de crear una institución especial dedicada a clarificar los procedimientos de privatización (Galal et., 94).

Sin embargo, tanto en el proceso de licitación, de otorgamiento de licencias, de regulación, y de operación en telecomunicaciones, los administradores extranjeros han encontrado pocas casos de corrupción en América Latina. Esto es especialmente así con respecto a empresas ya privatizadas. Sólo se han hecho llamados de atención con respecto a territorios donde el único sistema de telecomunicaciones existente es la empresa estatal. Pero, en general, observadores extranjeros han descrito las prácticas de negocios de la industria de telecomunicaciones latinoamericanas como por sobre la media mundial. 
Telecomunicaciones es, hoy en día, sinónimo de grandes negocios y alta política. En marzo de 1994, en la Conferencia Mundial de Desarrollo de las Telecomunicaciones, en Buenos Aires, Al Gore, Vicepresidente de los Estados Unidos, enfatizó el hecho de que las telecomunicaciones deberían figurar en la agenda política de cada país. En la misma ocasión, Pekka Tarjanne, Secretario General de la Unión Internacional de Telecomunicaciones, elaboró sobre la idea de ayudar a los países de menor desarrollo, ayudándoles a desarrollar las telecomunicaciones (Lynch, 94). En la economía global, donde las noticias acerca de los mercados financieros e innovaciones tecnológicas juegan un gran rol, las tecnologías de telecomunicaciones son un sector de primera importancia.

Un ejemplo de la naturaleza transnacional e internacional de las telecomunicaciones contemporáneas, es el Acuerdo Norte-Americano de Libre Comercio. La implementación de este tratado comercial requiere que los protocolos de transmisión, calidad, y disponibilidad de servicios sean uniformes en Canadá, los Estados Unidos, y México. Como caso, la compañía Southwestern Bell de los Estados Unidos tiene un especial interés en el mercado mexicano, pues su mercado territorial base bordea la frontera y los servicios de conexión, hacia y desde México, constituyen una proporción importante de sus negocios. En cuanto a tecnología se refiere, los troncales de la red de telecomunicaciones de México son lentos y no pueden soportar en forma adecuada la velocidad de transmisión requerida por los sistemas integrados de transmisión digital de voz/ dato/imagen. La necesidad de conectividad entre los sistemas de Canadá y los Estados Unidos ha sido lograda con la ayuda de un sistema de satélites. Pero a su vez, a diferencia de otras regiones en donde el sistema de satélites ha sido utilizado para eludir redes monopólicas, este sistema fue diseñado con el fin de expandir la capacidad del sistema mexicano.

Sería difícil argumentar que el desarrollo de las telecomunicaciones durante la última década ha sido negativo para los países latinoamericanos como un todo. Esto no significa que no existan problemas asociados a este desarrollo. Algunos observadores del mercado de telecomunicaciones latinoamericano predicen que la competencia va a moldear el mercado de manera tal, que en el futuro dominarán sólo un puñado de poderosas y verticalmente integradas redes. Peor, estas redes estarán controladas por gigantes de las telecomunicaciones de España, los Estados Unidos, e Italia. Es cierto que hay fuerzas orientando el mercado hacia grandes economías a escala. Por ejemplo, consolidando grandes 
paquetes de tráfico y enviándolos desde un solo punto de origen. Otros expertos, como Pekka Tarjanne, no creen que esa sea una acertada descripción del futuro (Pekka, 95). Ellos son de opinión de que la economía global incentivará el desarrollo de mercados locales, en donde las empresas tendrán que competir por acercar precios a costos decrecientes. La última conexión en la red, en el mercado local, piensan ellos, permanecerá como un lugar dominado por una variada gama de empresas más pequeñas y a menudo más eficientes, compitiendo entre sí y presionando a los gigantes. Esta diversidad crearía diferenciación de productos, innovación, y creatividad. Los acuerdos internacionales podrían estimular esta descentralización y coordinar la regulación de servicios a través de las fronteras. El acceso ilimitado a redes transfronterizas aseguraría la competencia en el mercado local.

Sin embargo, el libre flujo de tráfico telecomunicacional a través de las fronteras, o su transmisión a través del espacio extraterrestre, plantea numerosos problemas sensibles que transcienden el análisis técnico, económico, y comercial. Las dificultades para llegar a acuerdos de liquidación de pagos por conexión internacional son un claro ejemplo del peso que pueden tener consideraciones políticas en un hecho esencialmente técnico. Algunos países latinoamericanos, por ejemplo, prohíben cruzar la frontera con un teléfono celular, debido a una disposición legal que obliga a utilizar aparatos ensamblados localmente dentro de las fronteras del país en cuestión. Disposiciones de este tipo, ciertamente dificultan los acuerdos de liquidación de pagos y son un serio impedimento para el desarrollo del tráfico internacional. Con todo, la apertura de fronteras al tráfico telecomunicacional hemisférico es una proposición viable y cuyas eventuales ramificaciones económicas, comerciales, y culturales la transforman en un objetivo político extremadamente atractivo.

En el contexto de recientes empresas industriales surgidas en torno a nuevas tecnologías y creciente comercio entre las naciones, el hecho crucial es encontrar un balance entre regular para ordenar y evitar regulación que distorsiona los mercados y obstaculiza la innovación (Bhalla, 88). La industria de telecomunicaciones es un claro ejemplo de expansión dado los nuevos advances tecnológicos. Esta industria permite un fácil y barato flujo de información a nivel nacional e internacional. La nueva tecnología facilita un mejor entendimiento entre personas de diferentes culturas, pero también representa un peligro para la soberanía y seguridad nacional. Permite a las personas un acceso expedito a los medios de información, pero también facilita la intrusión en la privacidad. En definitiva, creemos que las telecomunicaciones avanzadas invitan a 
un renovado ejercicio en libertad y en prudencia de parte de los ciudadanos y de las naciones de Latinoamérica y del mundo.

\section{Conclusiones}

Dado el ambiente tecnológico contemporáneo, el gobierno de las telecomunicaciones mundiales es una tarea de proporciones gigantescas. Muchas de las dificultades se derivan de tres desarrollos importantes. Primero, la convergencia de dos tecnologías, computación y telecomunicación, está permitiendo un considerable progreso técnico y oportunidades para el desarrollo de servicios de telecomunicación digital multiconectados e interactivos. Segundo, la creciente independencia de la industria de telecomunicaciones del aparato estatal y del correspondiente gobiemo. Tercero, un cambio radical del mercado mundial ocasionado por el proceso de liberalización, diversificación y competitividad de la industria de telecomunicaciones.

Tal como ocurrió antes en la industria de computadores, las telecomunicaciones están en proceso de transición desde una industria con estándares técnico/comerciales pre-establecidos a una con estándares libres. Las empresas tradicionales deben adoptar nuevos estándares cada vez que las oportunidades de mercado lo justifiquen, aun cuando esto signifique baja de ingresos. Equipos tecnológicamente anticuados deben ser dados de baja en pocos años y no décadas. Adicionalmente, los monopolios de telecomunicaciones, que han sido dominados por una cultura ingenieril desde sus inicios, deben aprender ahora a pensar en términos de creación de valor agregado y servicio al cliente. Quizá la única sugerencia para prosperar en industrias de tecnología avanzada apunte a ser muy audaz y cauteloso simultáneamente.

Con relación a los gigantes de las telecomunicaciones, éstos no pueden suponer que habrá una victoria fácil en mercados emergentes como el latinoamericano. La penetración de nuevos mercados en economías emergentes es un buen objetivo. Pero, la lucha contra otros gigantes, por un lado, y nuevos operadores provenientes del mundo de la transmisión de datos y computación, por el otro, presenta dificultades enormes y grande será la tentación por alcanzar la mano protectora del gobierno local.

En presencia del nuevo mundo de Internet, de la transmisión de voz/ datos/imagen, y de la telecomunicación inalámbrica, los países latinoamericanos están obligados a seguir el tranco del avance tecnológico. Si no se hace ahora, se arriesga no poder hacerlo en el futuro. Este fue el 
principal estímulo para el cambio de política telecomunicacional y el consecuente impulso a las privatizaciones.

Incluso, especialistas en el área se han sorprendido por el rápido crecimiento de las telecomunicaciones en América Latina. Nuestra propuesta es que este fenómeno no es el resultado de una política específica, sino el resultado de una conjunción de al menos tres desarrollos. El primero tiene que ver con la evolución y avance de la tecnología misma. A mediados de los años sesenta, la evolución de la tecnología de telecomunicaciones tuvo un punto de inflexión que la acopló perfectamente a la tecnología computacional. Este fue un salto tecnológico que cambió la naturaleza de la tecnología y del negocio de la industria de telecomunicaciones. Significó mayor capacidad y mejor calidad a menor precio. Aún más, dada la naturaleza de esta nueva tecnología, comenzó a ser más fácil y económicamente eficiente, operar con redes totalmente nuevas, diseñadas con la nueva tecnología que modernizó las redes existentes. Este desarrollo tecnológico ha proporcionado una gran ventaja a empresas y economías emergentes.

El segundo desarrollo dice relación con la entrada de las compañías de telecomunicaciones al mercado global. El fenómeno de la globalización de la economía se originó sólo hasta cierto punto en el desarrollo de las telecomunicaciones. Es verdad que las telecomunicaciones son una infraestructura básica de la economía globalizada, pero ocurre que los gigantes de las telecomunicaciones habían sido fundamentalmente monopolios domésticos. Teniendo un mercado nacional cautivo, seguro, y estable, estas empresas habían sido extremadamente reacias "a salir a mar abierto". Fue la realidad de las nuevas tecnologías de telecomunicación, -un engendro que en parte ellas crearon-, debido, en gran parte, a factores ajenos a la industria de las telecomunicaciones y la globalización de la economía internacional, lo que forzó a estos gigantes a lanzarse a las aguas internacionales y a tratar de ser empresas globales.

El tercer desarrollo fue la introducción de políticas económicas de mercado en los países latinoamericanos. Dejando de lado las preferencias y diferencias ideológicas al respecto, la razón de estas políticas fue el objetivo de mejorar la eficiencia en la gestión de la economía. Este pensamiento calzó muy bien con las promesas de la nueva tecnología de telecomunicaciones y se vio en la industria de las telecomunicaciones la mejor oportunidad para un rápido caso exitoso. Es así como la privatización, liberalización y apertura a la competencia extranjera pregonadas por la política de mercado han avanzado más rápido en telecomunicaciones que en otras industrias latinoamericanas. 
En nuestra opinión, ninguno de estos desarrollos tecnológicos, políticos, económicos, y empresariales, independientemente considerados, pueden explicar el desarrollo de la industria de telecomunicaciones latinoamericana. Ciertamente, tampoco una específica política nacional o internacional de telecomunicaciones. Sin embargo, con el beneficio de la perspectiva histórica de los eventos, sí podemos explicar mejor el dramático cambio de esta industria apelando a la conjunción y acoplamiento de los distintos desarrollos mencionados.

El proceso político de la privatización de la industria de telecomunicaciones en América Latina puede asistir a los diseñadores de políticas en otras industrias y/o latitudes. La rápida y exitosa privatización en varios países latinoamericanos prueba que esta política es factible dada la voluntad y creatividad política. Cabe notar si que la privatización no es un proceso administrativo fácil y que enfrenta fuerte oposición. Los gobiernos deben diseñar una estrategia política que, por un lado, acoja las sofisticadas necesidades de telecomunicación de negocios con proyecciones internacionales, y por otra, las aspiraciones de sectores de bajos ingresos y/o apartados. Adicionalmente, la política debe contemplar la difícil tarea de negociar con quienes ven afectados sus intereses por la privatización.

El camino por delante no es nada fácil. Inadecuada inversión, en términos cuantitativos y cualitativos, falta de personal capacitado, pobres planes de mantención, políticas proteccionistas, rígidos mercados laborales, y el predominio de los más fuertes, sean multinacionales o bien organizados grupos de interés nacionales o internacionales, son todos factores que independientemente o en forma combinada y hasta conjunta pueden detener el objetivo de conectividad global para todos los latinoamericanos. Por último, pero muy importante, cabe mencionar que la implementación de la nueva industria de telecomunicaciones en América Latina es y será tan diversa como las naciones del continente. Por tanto, no existe un modelo latinoamericano de desarrollo de las telecomunicaciones que deba ser recomendado, sino un importante caso del cual aprender. 


\section{Bibliografía}

Bhalla, A. S. y James, D. (eds.), New Technologies and Development: Experiences in Technology Blending. (Boulder, CO: Lynne Rienner Publishers, 1988).

Boeker, Paul (ed.), Latin America's Turnaround Privatization, Foreign Investment, and Growth, (San Francisco, CA: ICS Press, 1993).

Ernst and Young, Privatization: Investing in State-Owned Enterprises Around the World, (New York, John Wiley and Sons, 1994).

Galal, Ahmed; Jones, Leroy; Tandon, Panjak y Volgelsand, Ingro, Welfare Consequences of Selling Public Enterprises, (New York: Oxford University Press, 1994).

Lynch, Karen y Preston, Robert. "Lessons in Change: The ITU debates Latin America's Liberalization Efforts as Part of an Examination of Worldwide Telecoms Development," LATimes on-line, $\mathrm{N}^{\mathrm{o}} 121,28$ de marzo de 1994, http:// techweb. cmp.com:80/techweb/prog.

Mody, Bella; Bauer, Johannes M. $\mathrm{y}^{\prime}$ Straubhaar, Joseph (eds.). Telecommunications Politics: Ownership and Control of the Information Highway in Developing Countries, (Mahwah, N.J.: Lawrence Erlbaum Associates Publishers, 1995).

Olufs, Dick W. The Making of Telecommunications Policy (Boulder, CO, Lynne Rienner Publisher, 1998).
Plaza, Óscar. "High-Technology in Societies in Transition", trabajo presentado en la reunión anual ISA/Midwest, Cleveland, Ohio, octubre de 1997.

Ramamurti, Ravi. (ed.), Privatizing Monopolies: Lessons from the Telecommunications and Transport Sectors in Latin America, (Baltimore y Londres: The Johns Hopkins University Press, 1996).

Roobeck, A.J., "Telecommunications: An Industry in Transition," en H. W. de Jong, (ed.), The Structure of the European Industry, (Dordrecht y Boston: Kluwer Academic Publisher, 1988).

Rowthorn, Bob y Ha-Joon Chang. "Public Ownership and the Theory of the State," en The Political Economy of Privatization, editado por Thomas Clarke y Christos Pitellis, (Nueva York: Routledge, 1993).

Storper, Nlichael; Thomadakis, Tavros B. y Tsipouri, Lena J., (eds.), Latecomers in the Global Economy, (Nueva York: Routledge, 1998).

Tarjanne, Pekka, "The GII: Moving Toward Implementation," Telecommunications, mayo de 1995. 